\title{
Development of ground pipeline system for high-level scientific data products of the Hisaki satellite mission and its application to planetary space weather
}

\author{
Tomoki Kimura $^{1, *}$, Atsushi Yamazaki ${ }^{2}$, Kazuo Yoshioka ${ }^{3}$, Go Murakami ${ }^{2}$, Fuminori Tsuchiya ${ }^{4}$, \\ Hajime Kita $^{2}$, Chihiro Tao ${ }^{5}$, Ichiro Yoshikawa ${ }^{3}$, Atsushi Kumamoto ${ }^{6}$, and Chisato Yamauchi ${ }^{7}$ \\ ${ }^{1}$ Frontier Research Institute for Interdisciplinary Sciences, Tohoku University, Aoba Ward, Sendai, Japan \\ ${ }^{2}$ Institute of Space and Astronautical Science, Japan Aerospace Exploration Agency, Sagamihara, Japan \\ ${ }^{3}$ Department of Complexity Science and Engineering, University of Tokyo, Kashiwa, Japan \\ ${ }^{4}$ Planetary Plasma and Atmospheric Research Center, Tohoku University, Sendai, Japan \\ ${ }^{5}$ National Institute of Information and Communications Technology, Tokyo, Japan \\ ${ }^{6}$ Space and Terrestrial Plasma Physics Laboratory, Tohoku University, Sendai, Japan \\ ${ }^{7}$ Misato Observatory, Kimino-cho, Wakayama, Japan
}

Received 13 July 2018 / Accepted 23 January 2019

\begin{abstract}
The Hisaki satellite is the first-ever space telescope mission dedicated to planetary sciences. Atmospheres and magnetospheres of our solar system planets are continuously monitored by the extreme ultraviolet (EUV) spectrometer onboard Hisaki. This paper describes a data pipeline system developed for processing high-level scientific and ancillary data products from the Hisaki mission. The telemetry data downlinked from the satellite are stored in a ground telemetry database, processed in the pipeline to imaging spectral data with a 1-min temporal resolution and ancillary data products, and then archived in a public database. The imaging spectra can be further reduced to higher-level data products for practical scientific use. For example, light curves of the power emitted from Jupiter's aurora and plasma torus with a temporal resolution of 10-min can be reduced from the imaging spectral data; the reduced light curves reveal the transport processes of energy and mass in Jupiter's magnetosphere and associated interplanetary solar wind conditions. Continuous monitoring with Hisaki will contribute considerably to our understanding of space weather relating to planets in our solar system.
\end{abstract}

Keywords: Hisaki / pipeline system / planetary magnetosphere / planetary atmosphere

\section{Introduction}

The extreme ultraviolet (EUV) space telescope, Hisaki, is the first small scientific satellite mission of the Japan Aerospace Exploration Agency (JAXA) to use a semi-custom-made bus system (Fukuda et al., 2008; Nakaya et al., 2011). The scientific scope of the mission is to investigate the dynamics of space and the atmospheric environments of planets in our solar system, such as the energy-mass transport in Jupiter's magnetosphere and the atmospheric escape from Venus and Mars (Yoshikawa et al., 2014). Furthermore, the concept of the mission is to provide a continuous monitoring platform for planets from the Earth-orbiting trajectory. The Hisaki satellite orbits in a

\footnotetext{
${ }^{*}$ Corresponding author: kimura@stpp.gp. tohoku. ac.jp
}

low-Earth orbit with an apogee of $1157 \mathrm{~km}$, a perigee of $954 \mathrm{~km}$ and an inclination of $29.7^{\circ}$. Since its launch on 14 September, 2013 to the present day (July 2018), Hisaki has observed Jupiter's magnetosphere for a total of 1000 days (approximately), which is an average of 200 observation days per year. Continuous long-term monitoring will enable studies of the auroral emissions of Jupiter, and it will be possible to determine how they are correlated with the "external" (associated with solar wind) or "internal" (associated with Io volcanoes) magnetospheric processes, as studied by Kimura et al. (2015, 2017, 2018). Hisaki enables long-term monitoring of the auroral emissions of Jupiter and is thus a unique observatory. Synergies with the Hubble Space Telescope and Juno spacecraft will also provide new insights into the physical processes operating in the polar magnetosphere of Jupiter. 
The onboard scientific instrument is the EXtreme ultraviolet spectrosCope for ExosphEric Dynamics (EXCEED) (Yoshioka et al., 2013), which comprises two subsystems: an EUV and a field-of-view guiding camera (FOV). In this paper we locally define the EUV wavelength as 10-150 nm, which overlaps the formal definition of the far ultraviolet (FUV) wavelength of $122-200 \mathrm{~nm}$.

The EUV instrument is a photon-counting spectrograph that measures the wavelength and arrival direction of each photon event utilizing three slits, a toroidal grating and a micro channel plate (MCP) with a resistive anode encoder (RAE), and the wavelength and arrival direction of the detected photons are integrated to produce two-dimensional (wavelength-space) spectral images. The toroidal grating and the three slits restrict the spectral range from $52 \mathrm{~nm}$ to $148 \mathrm{~nm}$ with a spectral resolution of $0.3-1 \mathrm{~nm}$ (depending on the wavelength). The angle of view ranges from -180 to +180 arcsec from the center of the FOV, a spatial plate scale of 4.1 arcsec/pixel and a spatial resolution of 17 arcsec are employed, and the effective area is typically $1-2 \mathrm{~cm}^{2}$ from $70 \mathrm{~nm}$ to $115 \mathrm{~nm}$. Details about the EUV instrument specifications can be found in Yoshioka et al. (2013).

The FOV camera guides the EUV instrument's FOV on the planet under observation. The FOV takes an image of the planetary body, which is transferred to a mission data processor (MDP), and the MDP analyzes the image to calculate the centroid of the planetary body. The centroid data are sent at a frequency of approximately $0.33 \mathrm{~Hz}$ to the satellite attitude control system (AOCS) for feedback control (Sakai et al., 2011), and feedback control then cancels blurring of images caused by thermal strain and shock from the body of the satellite. The in-flight performance test for feedback control by Yamazaki et al. (2014) indicated that the satellite pointing accuracy is successfully improved from 20-25 arcsec to 2 arcsec using feedback control.

The EUV and FOV instrument telemetry data are downlinked from the satellite to the ground station network and are stored in a telemetry database. We developed a ground pipeline that processes the raw data to produce the high-level data products used for scientific studies. These high-level data products are then published from the scientific data archive of JAXA to the internet, and metadata of the high-level data are also published from European metadatabases (see below).

\section{The JAXA data analysis network system (DANS)}

Hisaki's high-level scientific data products are generated in the JAXA DANS, which provides support for hardware and software and is shared by other space science missions. The DANS comprises the following subsystems: a telemetry database "SIRIUS", a pipeline processing server "Reformatter", a data archive and transmission system "DARTS" and associated data storage; the system is shown schematically in Figure 1, and the subsystems are further described as follows.

SIRIUS is a satellite telemetry database that contains archives of telemetry data, orbital element data and other data gathered during the science missions. SIRIUS stamps telemetry data from the data distribution system or from storage devices with a data creation time, and it provides the time-stamped telemetry as merged data. Reformatter provides a stable server environment to perform pipeline processing that creates highlevel science data for release via DARTS, and our pipeline system was developed in the Reformatter server (see Sect. 3 for details). DARTS (https://darts.jaxa.jp/) is a system used for distributing, searching and browsing scientific spacecraft data. The DARTS data archive provides resources on a wide range of space sciences, including astronomy, solar physics, solar-terrestrial physics and planetary sciences, and most of the data in the system originate from Japanese scientific satellites and explorers. DARTS is operated by the Center for Science-Satellite Operation and Data Archive (C-SODA, http://c-soda.isas. jaxa.jp/) at the Institute of Space and Astronautical Science (ISAS) of JAXA.

\section{Metadatabase}

As shown in Figure 1, metadata of Hisaki high-level data products (such as observation date, target name and observation mode) are archived in and published from European metadatabases, Virtual European Solar and Planetary Access (VESPA) (Erard et al., 2018; http://vespa.obspm.fr/planetary/ data) and Auroral Planetary Imaging and Spectroscopy (APIS) (Lamy et al., 2015; http://apis.obspm.fr/). VESPA and APIS are described as follows.

VESPA is run by the Europlanet 2020 Research Infrastructure programme, which built a Virtual Observatory (VO) for planetary sciences that connects all types of data and provides modern tools for retrieving, cross-correlating and displaying data and results of scientific analyses. Collaborating with Laboratoire d'Études Spatiales et d'Instrumentation en Astrophysique (LESIA) at the Observatoire de Paris, we developed a metadatabase server that is compatible with the VESPA VO and is used to archive metadata from Hisaki high-level data in the server. Users can search for Hisaki data on the web interface of VESPA and download quicklooks and high-level data from DARTS via the interface. The APIS service aims to provide free and simple access to processed, high-level and auroral data of planets. APIS was developed at LESIA, the Observatoire de Paris and the Centre National de la Recherche Scientifique and is maintained by the Paris Astronomical Data Centre. Users can also search for Hisaki data on the APIS web interface and download data from DARTS via the interface.

\section{Pipeline system in Reformatter}

\subsection{System overview}

The pipeline system is developed in the Reformatter server, which is a Red Hat Enterprise Linux environment, and data processing software tools are coded in $\mathrm{C}, \mathrm{C}++$ and Interactive Data Language (IDL). Science telemetry data are transferred from the telemetry database SIRIUS to Reformatter to create scientific data products for the EUV and FOV instruments in NASA's FITS format (e.g., https://fits.gsfc.nasa.gov/), and each of the data products is defined in Section 4.2. Furthermore, FITS data are manipulated with a new FITS Input/Output library for next generation, SFITSIO, version 1.4 developed by Chisato Yamauchi (http://www.ir.isas.jaxa.jp/ cyamauch/). 


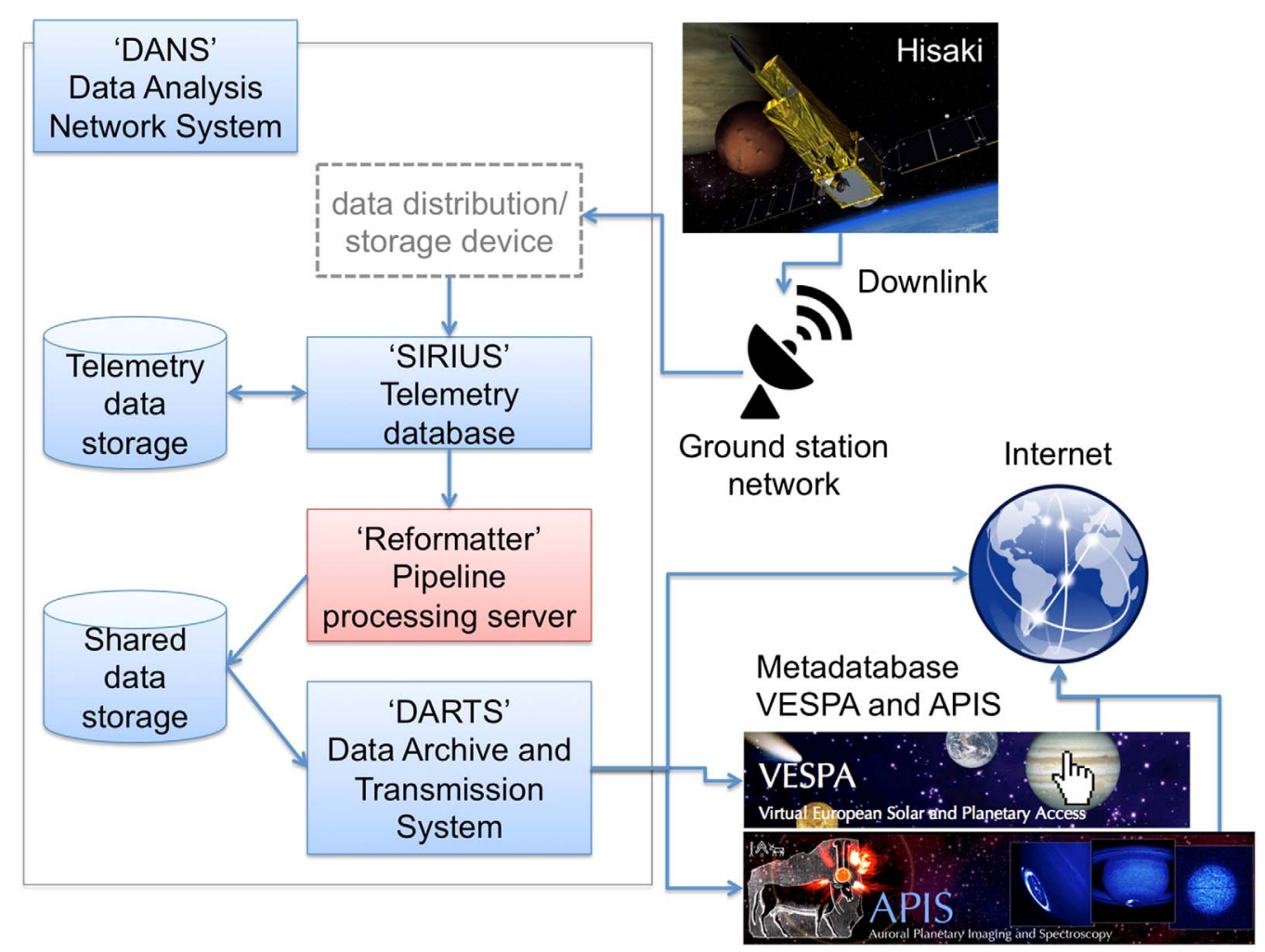

Fig. 1. Overview of Data Analysis Network System (DANS). Telemetry data downlinked from Hisaki are received at the ground station network and stored in the data distribution/storage device. The pipeline system processes the stored telemetry data transferred via the telemetry archive SIRIUS. The processed data are archived as high-level science data products in the Data Archive and Transmission System (DARTS), and high-level data products are published to the internet from DARTS. Metadata of the high-level data are archived in European metadatabases, VESPA and APIS, and are published to the internet.

Ancillary data, such as the satellite trajectory, attitude, spacecraft event time and instrument and satellite coordinates, are in the SPICE kernel format defined by NASA Navigation and Ancillary Information Facility (NAIF, https://naif.jpl.nasa. gov/). Using a telemetry manipulation library, L1TSD SPICE version 1.03 developed by C-SODA, we generate Hisaki SPICE kernels from Hisaki housekeeping (HK) telemetry, orbital elements and the leap second table provided from JAXA. HK telemetry that are not associated with the SPICE kernels are processed with the L1TSD TLM2FITS library version 2.03 and attached as header information in the EUV and FOV FITS file. The time codes of science and HK telemetry data, which correspond to the telemetry packet creation time on the satellite, are calibrated to universal time by the JAXA time-tag library, and both the L1TSD and time-tag libraries are distributed from JAXA/C-SODA.

An overview of the pipeline system is shown in Figure 2, and the processing of each data product is described in Sections 4.3-4.7.

\subsection{Definition of high-level data products}

The EUV instrument products are categorized into three levels: EUV-L0, -L1 and -L2, and Figure 3 illustrates the FITS file structures of each product. EUV-L0 provides a time series of observed photon event times and four uncalibrated voltages corresponding to the location of the photon on the EUV instru- ment detector. EUV-L1 provides a time series of photon events with the arrival time, wavelength and arrival direction of observed photons; the wavelength and arrival direction are calculated with calibration data based on the methods described in Section 5.1. EUV-L2 provides a time series of two-dimensional (wavelength-space) imaging spectral data that are reduced by the temporal integration of EUV-L1. The integration time of each imaging spectrum is typically $60 \mathrm{~s}$. The imaging spectra of EUV-L2 are the photon count rate distributions, from which users estimate absolute brightness in Rayleigh/pixel utilizing the brightness calibration data EUV-CAL. See Section 5.2 for the brightness calibration method. Furthermore, EUV-L2 and EUV-CAL data are distributed from DARTS.

FOV-L0 and -L1 are time series of two-dimensional (spacespace) images of planetary bodies. Integration times for each image are typically $0.6-1 \mathrm{~s}$, and these are selected with respect to the observation target. The temporal interval between each downlinked image is typically $130 \mathrm{~s}$. FOV-L0 comprises the image data extensions, while FOV-L1 additionally includes ancillary information on observations, such as planetary ephemeris, spacecraft location and attitude (see Fig. 4). FOV-L1 is archived and published in DARTS.

See the data format definition document at https://darts.jaxa. $\mathrm{jp} /$ pub/hisaki/doc/ for details of EUV and FOV data products.

Seven types of SPICE kernels are generated in the pipeline system, with which the SPICE toolkits provide the spacecraft trajectory, attitude, universal time, instrument and satellite 


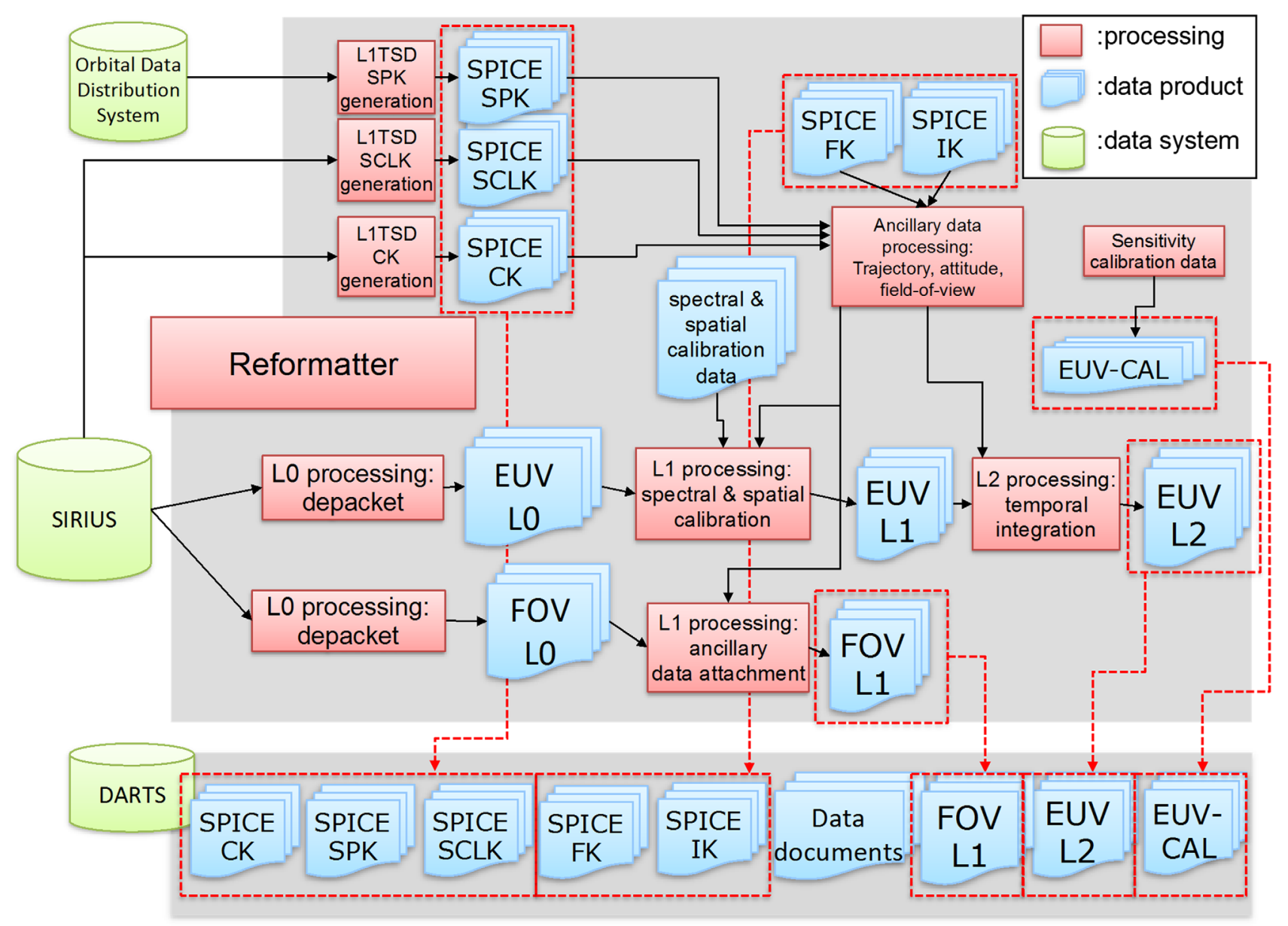

Fig. 2. Overview of pipeline system in Reformatter. See Section 4.2 for the definition of each data product, and Sections $4.3-4.7$ for each of the data processing routines. The orbital data distribution system outside the DANS conveys the Hisaki trajectory data to Reformatter.

coordinates, leap seconds and planetary parameters. The format of the kernels is compliant with that defined by NASA/NAIF, and the SPICE kernels are distributed from DARTS.

The scientific and ancillary data products introduced above are summarized in Tables 1-3.

\subsection{EUV-LO processing}

The SIRIUS database stores telemetry packets of the science instrument and satellite HK data, which are transferred to the Reformatter server on demand from the pipeline system. The transferred telemetry is depacketed in EUV-L0 processing, and raw photon-counting data of the EUV instrument is extracted from the depacketed telemetry. Figure 5 shows a raw photon-counting data series. Each photon event comprises 8 bytes of data ( 2 bytes $\times 4$ channels) that are output voltages from the four corners of the rectangular shaped EUV detector. A set of the 4-channel voltages corresponds to a photon location on the detector. An 8-byte satellite time code, of which first 4-bytes are actually padded with 0xFFFF in hexadecimal, is inserted into the series of the photon events every second. At most, 10000 photon events are measured every second and downlinked to the ground.
In the EUV-L0 processing stage, each photon event and corresponding 4-channel voltages are tagged with a time code using a time resolution of $1 \mathrm{~s}$; therefore, every photon detected within each $1 \mathrm{~s}$ interval has the same time code. Time series of time-tagged photon events are stored in the binary table extension known as "Event", as shown in Figure 3. The time series of $\mathrm{HK}$ data at the photon event time are also depacketed from HK telemetry received from SIRIUS and are then attached as an additional binary table extension in the EUV-L0 FITS file and used for the downstream processing. Each EUV-L0 FITS file spans an observation time of $1 \mathrm{~d}$. The maximum exposure time is $12 \mathrm{~h}$ /day, and the observable maximum photon count rate is $1000 \mathrm{~Hz}$. Thus, the estimated maximum daily photon count is approximately 43 million, which equates to approximately 6 GB of data volume. However, the actual daily photon count rate is much lower: for example, 3-5 million for Jupiter, which uses a total data volume of only a few $100 \mathrm{MB}$.

\subsection{EUV-L1 processing}

The event time and photon location on the detector are recorded in arbitrary units in EUV-L0 data. Therefore, in EUV-L1 processing, we calibrate the arbitrary values to the 
EUV-LO

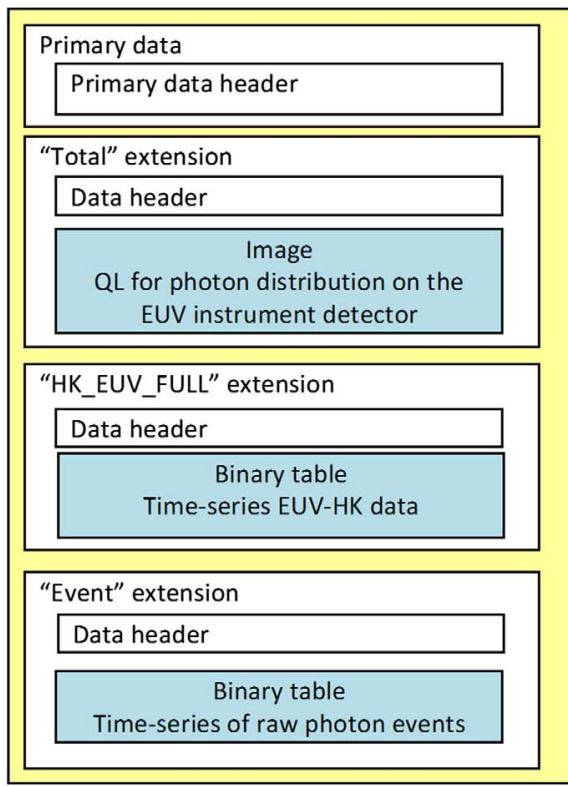

EUV-L1

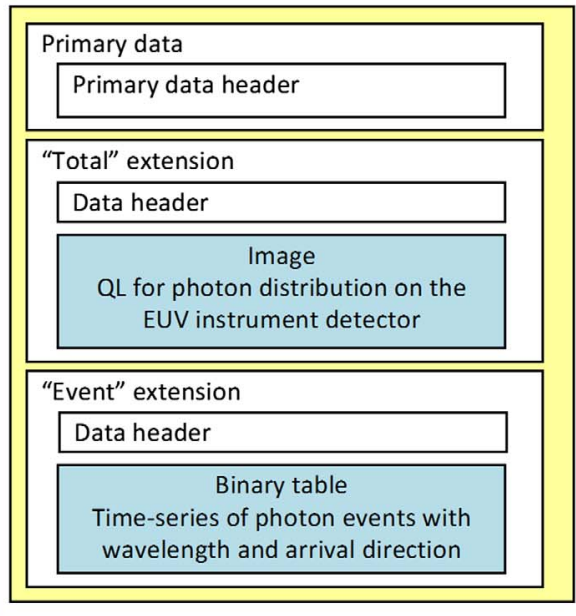

EUV-L2

\begin{tabular}{|c|}
\hline Primary data \\
\hline Primary data header \\
\hline "Total" extension \\
\hline Data header \\
\hline $\begin{array}{l}\text { Image } \\
\text { QL for photon distribution on the } \\
\text { EUV instrument detector }\end{array}$ \\
\hline “YYYY-MM-DDThh:mm:ss" extension \\
\hline Data header \\
\hline $\begin{array}{l}\text { Image } \\
\text { "YYYY-MM-DDThh:mm:ss" } \\
\text { spectral imaging data }\end{array}$ \\
\hline "YYYY-MM-DDThh:mm:ss" extension \\
\hline Data header \\
\hline $\begin{array}{c}\text { Image } \\
\text { "YYYY-MM-DDThh:mm:ss" } \\
\text { spectral imaging data }\end{array}$ \\
\hline
\end{tabular}

Fig. 3. FITS file structures of EUV-L0, -L1 and -L2. In addition to the scientific data extension ("Event" in EUV-L0 and -L1 and "YYYY-MMDDThh:mm:ss" in EUV-L2), each file contains a primary header and a quick look (QL) image of photon distribution on the EUV instrument detector. EUV-L0 includes time series of the HK data used for downstream processing.

\section{FOV-L0 \& L1}

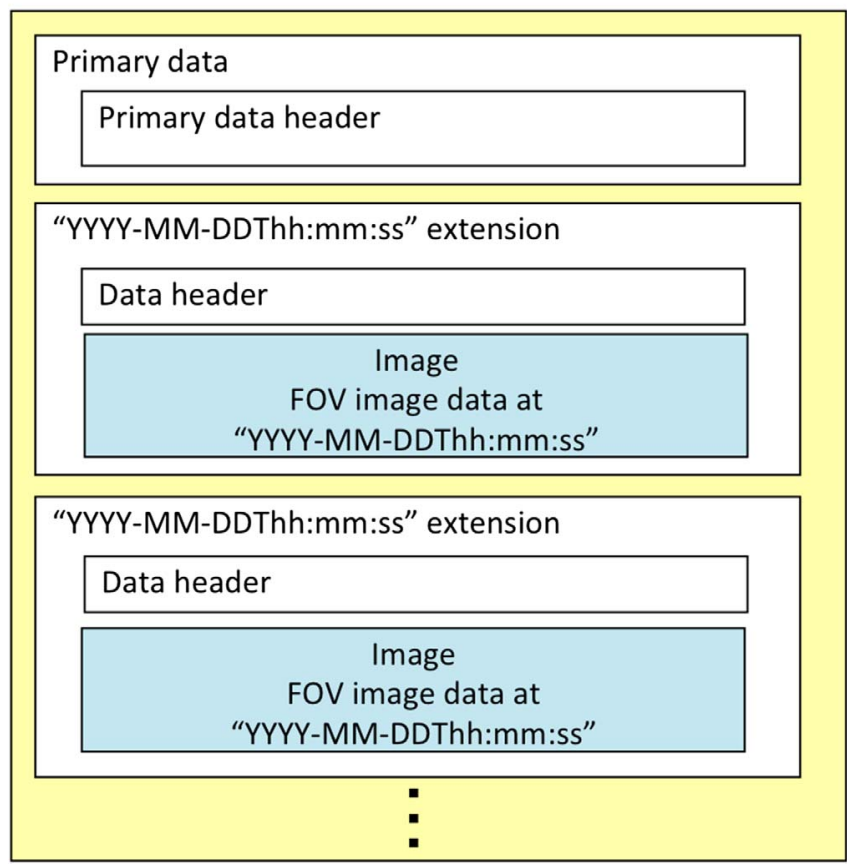

Fig. 4. FITS file structures of FOV-L0 and L1. In addition to the scientific data extension "YYYY-MM-DDThh:mm:ss" (see text for detailed contents), the file stores a primary data header.

universal time, wavelength and arrival direction of each photon event, and a time series of the universal time, wavelength and arrival direction are stored in the binary table extension "Event" of the EUV-L1 FITS file (Fig. 3). See Section 5.1 for the calibration methods of wavelength and arrival direction. Some ancillary data, such as the observation target, spacecraft trajectory and instrument FOV, are computed with SPICE kernels and other HK data, and they are then attached as a header to the binary table extension. The EUV-L1 FITS files are organized by target, observation mode and observation date, and the total data volume of an EUV-L1 FITS file is typically a few $100 \mathrm{MB} / \mathrm{d}$.

A fraction of the observed photon events is removed when the EUV detector is unable to correctly measure the 4-channel voltages; this occurs either as a result of probabilistic errors in the instrument or with respect to system electronics and detector gain characteristics. These "error" values are randomly distributed in the binary table. In common FITS manipulation methods, error values in a data array are removed by copying rows and resizing the array for each error value. However, such methods incur huge computing costs, particularly with respect to resizing the array, as millions of rows in the array have to be resized for each error value. Therefore, we use a computing-cost-saving algorithm to remove the error rows, as shown in Figure 6. In the first processing of Figure 6, a row with an error value of ERR is overwritten with the following normal values $y-z$. After overwriting all the error values, the array is resized only once, and this dramatically reduces the computing cost.

\subsection{EUV-L2 processing}

The EUV-L2 data are time series of two-dimensional (wavelength-space) imaging spectra integrated with a fixed exposure time of typically $60 \mathrm{~s}$. The $60 \mathrm{~s}$ time series of photon events are binned to a 2D photon count histogram of 1024 pixels 
Table 1. Product list for EUV instrument data.

\begin{tabular}{ll}
\hline Data product & Definition \\
\hline EUV-L0 & Time series of raw data of photon event detected by the EUV instrument \\
EUV-L1 & Time series of photon event with wavelength, and arrival direction reduced form EUV-L0 \\
EUV-L2 & Time series of two-dimensional imaging spectral data reduced from EUV-L1 \\
EUV-CAL & two-dimensional image data of wavelength, arrival direction, and sensitivity used for EUV-L2 calibration \\
\hline
\end{tabular}

Table 2. Product list for the FOV instrument data.

\begin{tabular}{ll}
\hline Data product & Definition \\
\hline FOV-L0 & Time series of two-dimensional image of planetary shape measured with the FOV instrument \\
FOV-L1 & The same product as FOV-L1, but with ancillary data (e.g., spacecraft trajectory and planetary ephemeris) \\
\hline
\end{tabular}

Table 3. Product list for the SPICE kernels.

\begin{tabular}{ll}
\hline Data product & Definition \\
\hline Trajectory Kernel (SPK) & Trajectories of spacecraft and solar system bodies \\
Orientation and Attitude Kernel (CK) & Attitude and orientation of spacecraft \\
Spacecraft Clock Kernel (SCLK) & Calibration of spacecraft instrument coordinates \\
Frame Kernel (FK) & Definition of spacecraft coordinates \\
Leapseconds Kernel (LSK) & List of leapseconds \\
Planetary Constant Kernel (PCK) & Orbital elements and shape of planets \\
\hline
\end{tabular}

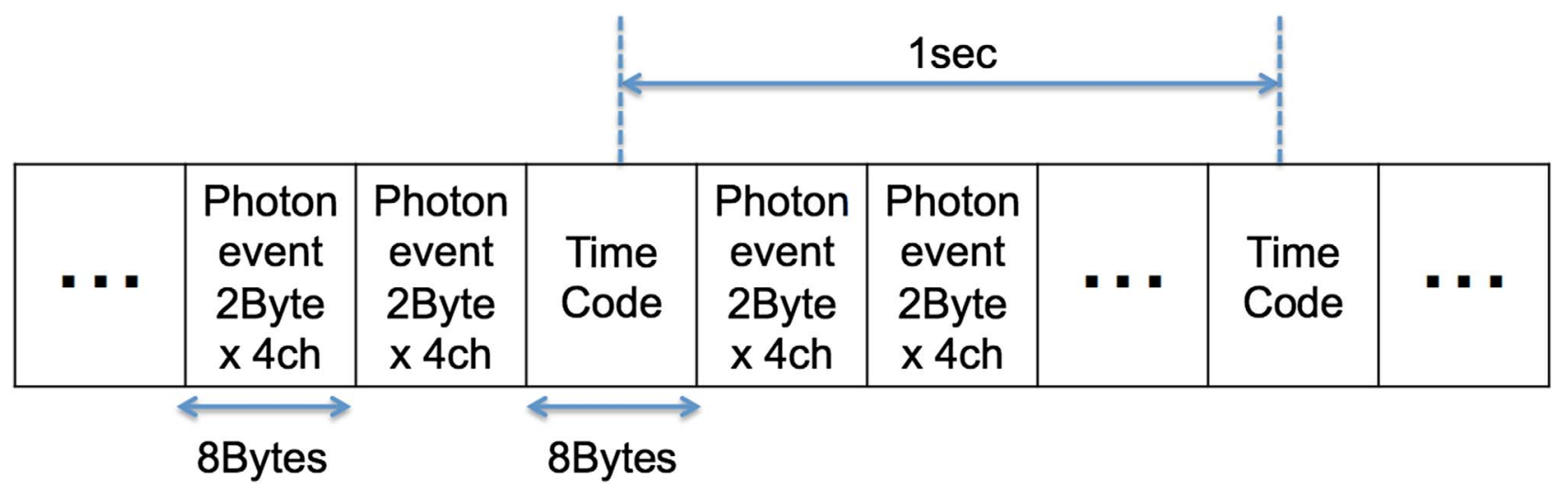

Fig. 5. Raw photon-counting data of EUV instrument.

(wavelength) $\times 1024$ pixels (arrival direction), and this temporal integration is applied for all photon events in the EUV-L1 file. Spatial plate scales are approximately 4.1 and 9.8 arcsec/ pixel in the spatial and wavelength axes, respectively, and one pixel along the wavelength axis corresponds to approximately $0.11 \mathrm{~nm}$. In the EUV-L2 FITS file, a 2D imaging spectrum is stored as an image extension "YYYY-MM-DDThh:mm:ss", which corresponds to the start time of the integration. The image size is 32 bits $\times 1024$ pixels $\times 1024$ pixels, i.e., $4.2 \mathrm{MB}$. Some ancillary data are computed from the SPICE kernels and other HK data, and they are then attached as the header information to each image extension. Each EUV-L2 data file comprises a set of image extensions for observations obtained during 1 day, typically around 600-700 images, which are organized by the target and observation mode, and the total data volume of an EUV-L2 FITS file is typically 1-3 GB. Figure 7 shows an example of EUV-L2, which was observed on 1 February,
2015, where the observation targets were Jupiter's aurora and the Io plasma torus.

Absolute brightness (Rayleigh/pixel) was calculated by multiplying a two-dimensional coefficient array of the EUV-CAL with the EUV-L2 imaging spectrum. See Section 5.2 for derivation of the coefficient of EUV-CAL.

\subsection{FOV-LO and L1 processing}

The FOV instrument captures the shape of planetary bodies with a CCD camera that uses a fixed exposure time (typically $0.6-1 \mathrm{~s})$. An image that has 16 bits $\times 256$ pixels $\times 256$ pixels $(\sim 131 \mathrm{~KB})$ is generated every $3 \mathrm{~s}$, and this is sent to the MDP subsystem to compute the centroid of the planetary shape. Typically, every $130 \mathrm{~s}$, a $3 \mathrm{~s}$ exposure image is subdivided into packets with a size of roughly $1 \mathrm{~KB}$, stored in the satellite data recorder and then downlinked to the ground. 


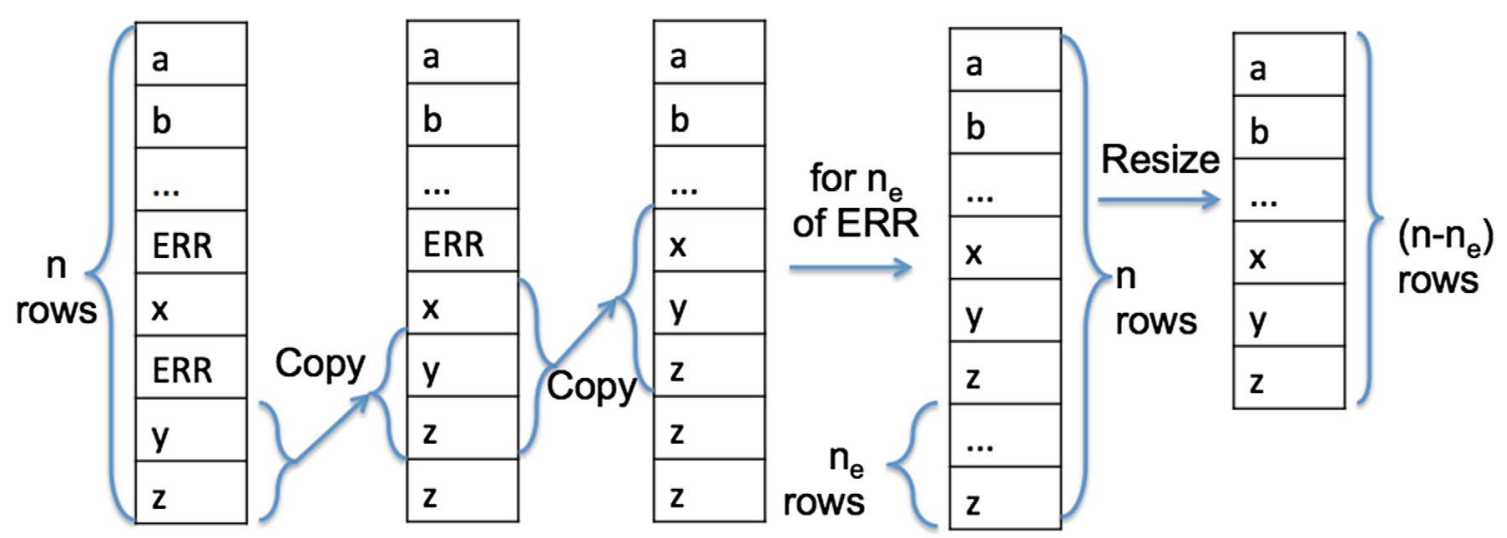

Fig. 6. Algorithm for removing error values in the binary table extension. The number, $n_{\mathrm{e}}$, of error values are randomly distributed in the data array, which has millions of rows. In the first step of processing, a row with an error value ERR is overwritten with the following normal values $y-z$. After overwriting all error values, the data array is resized only once, and $n_{\mathrm{e}}$ rows are removed from the end of the array.

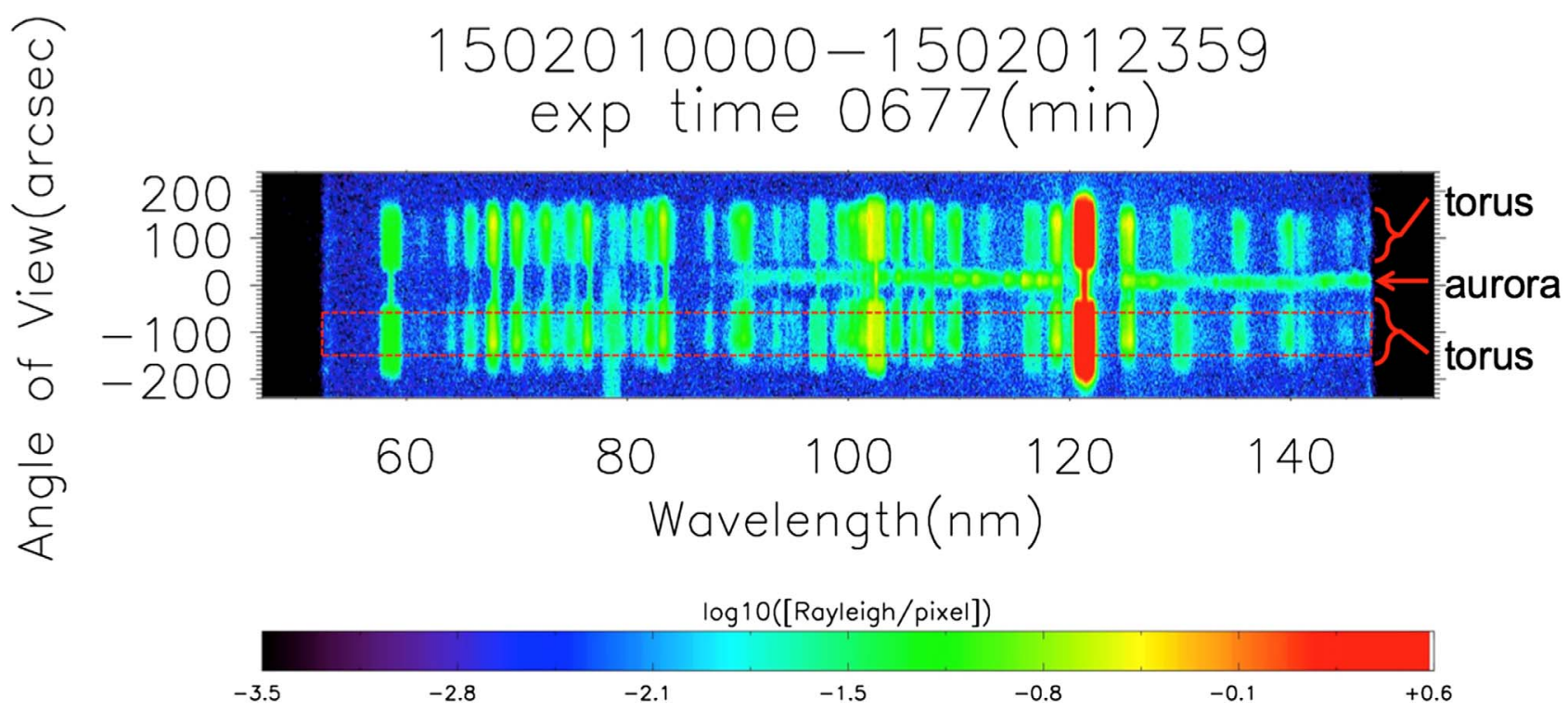

Fig. 7. Integrated imaging spectrum of EUV-L2 observed on 1 February, 2015. Horizontal and vertical axes show wavelength (nm) and arrival direction $(\mathrm{arcsec})$ of photon events, respectively. Color shows the brightness in Rayleigh/pixel in logarithmic scale. This figure plots the region of 1024 (wavelength axis) $\times 117$ (spatial axis) pixels on the detector for good visibility. Observation targets were Jupiter's aurora and the Io plasma torus. Spatial regions corresponding to the aurora and torus are annotated by the red arrow and curly brackets, respectively. Integration time is 677 min i.e., 677 image extensions of EUV-L2, corresponding to a 1-day observation time. Brightness in the region framed with the red broken line is reduced to the one-dimensional spectrum in Figure 11 by spatial integration. The framed region corresponds to a planeto-centric distance of 3-7 Jovian radii from Jupiter.

In the pipeline system, the FOV telemetry packets transferred from SIRIUS are depacketed, from which the FOV image is reconstructed. The reconstructed FOV images are stored as image extensions in the FOV-L0 FITS file, and all images taken on 1 day are stored as image extensions in the same FOV-L0 FITS file. Each extension includes an image with a data size of 32-bit integers (counts) $\times 256$ pixels $\times 256$ pixels, which is equivalent to approximately $260 \mathrm{~KB}$. The maximum number of images is 9 images every $130 \mathrm{~s}$ for $12 \mathrm{~h} / \mathrm{d}$, or 2990 images per/d. The FOV-L1 FITS file is in the same format as FOV-L0, but, some ancillary data, such as observation target, spacecraft trajectory and instrument FOV, are computed with the SPICE kernels and other HK data, and they are then attached as header information on each image extension. Each FOV-L1 data file comprises a set of image extensions for observation obtained during 1 day (typically around 600-700 images) with a total volume of $\sim 200 \mathrm{MB}$, and these are organized by the target and observation mode. Figure 8 shows an example of FOV-L1, which was taken for Jupiter on a February, 2015, which is the same observation date as that used in Figure 7.

\subsection{SPICE kernel processing}

Using the L1STD SPICE library, the trajectory kernel (SPK) is generated from the satellite trajectory data provided by the JAXA Orbital Data Distribution System. In orientation and 


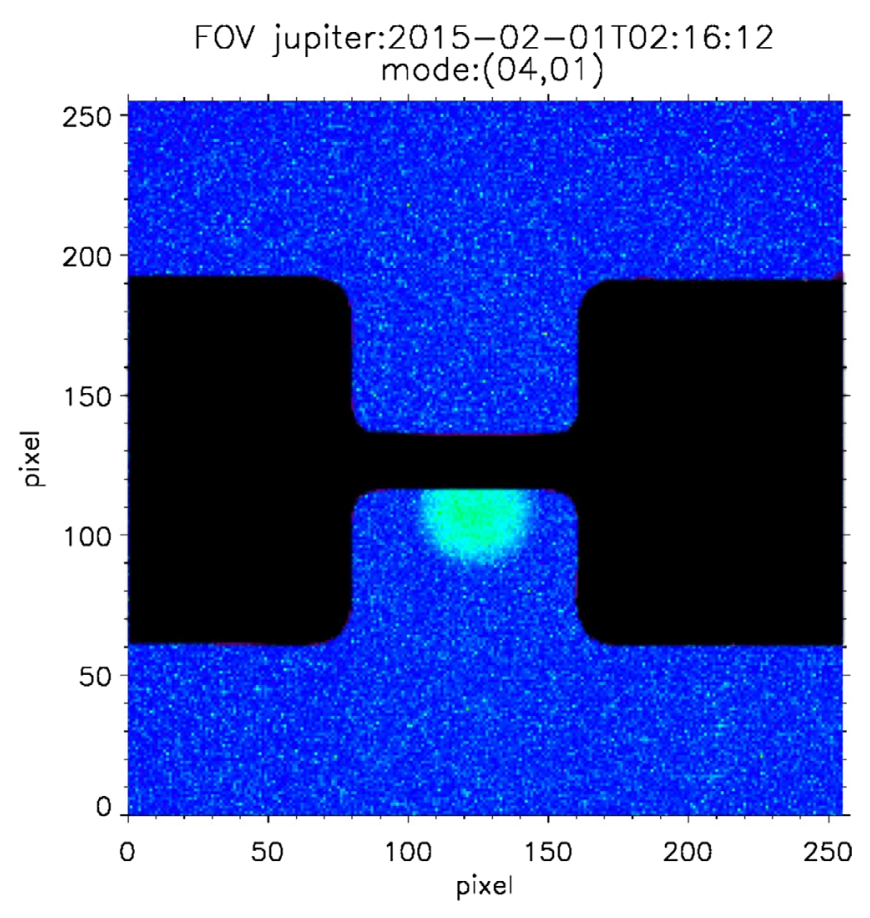

Fig. 8. Imaging data of FOV-L1 observed on 1 February, 2015. Horizontal and vertical axes show spatial locations in the detector coordinates (pixel). The horizontal and vertical axes roughly align with east-west and north-south directions of the ecliptic coordinates, respectively. Plate scale is 1.1 arcsec/pixel. Color shows arbitrary photon counts. Observation target was Jupiter, the shape of which is indicated in light green. The solid black region illustrates the expected location of the dumbbell-shaped slit of the EUV instrument, which is actually not seen in FOV data because the count rates of the sky background and dumbbell slit region are both at the noise level of the FOV detector. Integration time is $1 \mathrm{~s}$.

attitude kernel (CK) processing, the L1TSD SPICE library receives HK telemetry packets of satellite attitude data from SIRIUS; it then depackets these and stores them in a kernel file in the CK format. The spacecraft clock kernel (SCLK) is generated from the Satellite Time Calibration Data File distributed from SIRIUS; this associates the satellite time code, which is recorded in an arbitrary unit, with universal time. We manually wrote the instrument and frame kernels (IK and FK) in an ASCII format to define the instrument and spacecraft coordinates. The IK and FK kernels associate the boresight vector and FOV of the instrument with the satellite coordinates. The JAXA-defined leap second list is reformatted in the leap seconds kernel (LSK) with L1TSD SPICE. The planetary constant kernel (PCK) is downloaded to Reformatter from the NASA/NAIF web site.

\section{Data calibration}

\subsection{Calibration of wavelength and arrival direction of photon}

As described by Yoshioka et al. (2013), in the EUV instrument, an incoming photon spectrally dispersed with toroidal grating interacts with the surface of MCP; this initiates an electron cascade, and electron clouds formed by the cascade are detected by the RAE as an electric current. The electric current is collected at the four corners of the RAE, and the voltage pulse heights are measured and digitized as photon event data. The 4-channel voltages recorded at the corners of the RAE correspond to the location of the photon event on the RAE, and they indicate the wavelength and arrival direction. The 4-channel voltages, wavelength and arrival direction are related by

$$
\begin{aligned}
& x=a_{x} \frac{V_{1}+V_{2}}{V_{1}+V_{2}+V_{3}+V_{4}}, \\
& y=a_{y} \frac{V_{2}+V_{3}}{V_{1}+V_{2}+V_{3}+V_{4}},
\end{aligned}
$$

where $x$ is the wavelength in pixels of the wavelength axis; $y$ is the arrival direction in pixels of the spatial axis; $V_{1}, V_{2}, V_{3}$ and $V_{4}$ are the measured 4-channel voltages at the RAE corners in 12-bit number; and $a_{x}$ and $a_{y}$ are scaling factors for the wavelength and spatial axis, respectively, which are set as 1024 pixels.

Gain (in pC) for each RAE channel is also computed from the 4-channel voltages by the following relation,

$$
\text { Gain }=0.5 \times \frac{V-P_{1}}{P_{2}},
$$

where $V=V_{1}, V_{2}, V_{3}, V_{4}$ and $P_{1}$ and $P_{2}$ are correction factors defined in Table 4 that were estimated from the ground test. If the gain exceeds $3.5 \mathrm{pC}$, it is not possible to correctly calibrate the detected photon location on the detector because of the design of the instrument's electronics. Events with gains greater than $3.5 \mathrm{pC}$ are discarded for calibration of wavelength, arrival direction and downstream processing (see Murakami et al., 2010 and Yoshikawa et al., 2014 for details on the detector gain).

Spectral lines have an inclination in the wavelength-space plane that are associated with an alignment error of the optical system, and the inclination is corrected with the following relations,

$$
\begin{aligned}
& x^{\prime}=x-(x-512) \tan \alpha, \\
& y^{\prime}=y-(y-512) \tan \beta,
\end{aligned}
$$

where $x^{\prime}$ and $y^{\prime}$ are the corrected wavelength and arrival direction in pixels, and $\alpha=-0.48^{\circ}$ and $\beta=-0.49^{\circ}$ are the angles for the correction, which were evaluated from the ground test. The absolute wavelength, $\lambda(\mathrm{nm})$, of the photon event is calibrated with a fourth-order polynomial function for the $x^{\prime}$ th pixel,

$$
\lambda=c_{0}+c_{1} x^{\prime}+c_{2} x^{\prime 2}+c_{3} x^{\prime 3}+c_{4} x^{\prime^{4}},
$$

where the coefficients from $c_{0}$ to $c_{4}$ are defined in Table 5 and were estimated from flight calibration based on the geocoronal emission observation made on 29 November, 2013.

The absolute arrival direction, $r$ (arcsec), is calibrated with a linear function for the $y^{\prime}$ th pixel as

$$
r=p_{y}\left(y^{\prime}-y_{0}\right)
$$


Table 4. Correction factors for EUV instrument gain calibration.

\begin{tabular}{lcc}
\hline Channel & $\mathrm{P}_{1}$ & $\mathrm{P}_{2}$ \\
\hline $\mathrm{V}_{1}$ & 2 & 1058 \\
$\mathrm{~V}_{2}$ & 3 & 1099 \\
$\mathrm{~V}_{3}$ & 2 & 1088 \\
$\mathrm{~V}_{4}$ & 4 & 1072 \\
\hline
\end{tabular}

Table 5. Coefficients for wavelength calibration estimated from observation of Earth's geocorona on 29 November, 2013.

\begin{tabular}{lc}
\hline Coefficient & Value \\
\hline $\mathrm{c}_{0}$ & +152.94 \\
$\mathrm{c}_{1}$ & -0.093260 \\
$\mathrm{c}_{2}$ & $-4.9140 \times 10^{-5}$ \\
$\mathrm{c}_{3}$ & $+6.2555 \times 10^{-8}$ \\
$\mathrm{c}_{4}$ & $-2.3824 \times 10^{-11}$ \\
\hline
\end{tabular}

where $p_{y}$ is the plate scale of $4.1 \mathrm{arcsec}$ in the spatial axis, and $y_{0}$ is the center of the slit at pixel 572; these values were also estimated from the geocoronal emission observation conducted on 29 November, 2013. The calibration coefficients for the wavelength and plate scale are dependent on time and are calibrated based on geocoronal observations roughly annually. See Yoshioka et al. (2013) for more details on the wavelength and arrival direction calibration.

\subsection{Calibration for brightness}

In the EUV-L2 processing, time series of $\lambda$ and $r$ with an exposure time of $60 \mathrm{~s}$ are binned to a two-dimensional wavelength-space histogram of $1024 \times 1024$ pixels. The integrated imaging spectrum is the distribution of the photon count rate. At the $i$ th wavelength pixel in any spatial pixel row, the photon count rate, $C_{i}$ (in units of counts $/ \mathrm{min} / \mathrm{pixel}$ ), is calibrated to the absolute brightness, $B_{i}$ (in Rayleigh/pixel) (Rayleigh: $10^{6} / 4 \pi$ photons steradian ${ }^{-1} \mathrm{~cm}^{-2} \mathrm{sec}^{-1}$ ) utilizing the following relation,

$$
\begin{gathered}
B_{i}[\text { Rayleighpixel }] \sim C_{i}[\text { counts } / \text { min pixel }] /\left[\frac{10^{6}[\text { counts }]}{4 \pi[\mathrm{sr}]}\right. \\
\times 60[\mathrm{~s}] \times\left(\frac{\pi[\mathrm{rad}]}{180^{\circ}} \frac{4.1[\mathrm{arsec}]}{3600[\mathrm{arsec}]}\right) \times\left(\frac{\pi[\mathrm{rad}]}{180^{\circ}} \frac{9.8[\mathrm{arsec}]}{3600[\operatorname{arsec}]}\right) \\
\left.\times A_{i}\left[\mathrm{~cm}^{2}\right]\right] \sim C_{i} /\left(4.51 \times 10^{-3} A_{i}\right)
\end{gathered}
$$

where $A_{i}$ is the effective area of the detector at the $i$ th wavelength pixel. Here we assume an isotropic photon emission from the target; its brightness is distributed uniformly in a pixel with a 4.1 arcsec (spatial axis) $\times 9.8$ arcsec (wavelength axis) viewing angle, and there is no dependence on any of the parameters in Equation (8) on the photon arrival direction. It is of note that there are actually $\sim 10 \%$ spatial variabilities in the effective area; these were estimated from observations of the terrestrial geocoronal emission, which is an approximately flat brightness source observed within the viewing angle of the slit (360 $\operatorname{arcsec} \times 140 \operatorname{arcsec})$. The absolute value of the effective area, $A_{i}$, was estimated by in-flight calibration based on observations of a spectrophotometric standard star: for example, the white dwarf GD71 observed on 29 July, 2016 was used to calibrate observation data from 1 June, 2015 to the present day (July 2018), as shown in Figure 9. Note that below $93 \mathrm{~nm}$ there is strong absorption of EUV photons by the interstellar medium between GD71 and Hisaki, which dims the signal out. Therefore $A_{i}$ below $93 \mathrm{~nm}$ is estimated by multiplying the ground calibration data with a factor that connects $A_{i}$ smoothly with that above $93 \mathrm{~nm}$. See Yoshikawa et al. (2014) and Yoshioka et al. (2013) for more details on the brightness calibration.

The calibrated $\lambda, r$ and coefficient $1 /\left(\left(4.51 \times 10^{-3} A_{i}\right)\right)$ are stored in the EUV-CAL FITS file, as shown in Figure 10. These quantities are expanded to $2 \mathrm{D}$ images with $1024 \times 1024$ pixels and stored as the "X-coord", "Y-coord" and "Cal" extensions, respectively.

\section{Case-study of Hisaki data product usage}

\subsection{Data reduction for light curve}

For practical scientific use, the EUV-L2 product can be further reduced to certain higher-level products by users. For example, 1D spectra with high signal-to-noise ratios reduced from EUV-L2 were used for spectral diagnostics and ion line identification for the Io plasma torus by Yoshioka et al. (2014, 2017, 2018) and Hikida et al. (2018). See Figure 11 for an example of the reduced 1D spectrum.

The light curve of brightness or emission power is one of the most useful products, and it has been used in almost all Hisaki monitoring studies, such as Jupiter's aurora (Kimura et al., 2015, 2016, 2017, 2018; Badman et al., 2016; Gray et al., 2016; Tao et al., 2016a,b, 2018; Kita et al., 2016; Nichols et al., 2017), the Io plasma torus (Tsuchiya et al., 2015, 2018; Murakami et al., 2016; Yoshikawa et al., 2016, 2017; Suzuki et al., 2018), Io's atmosphere (Koga et al., 2018a,b), the Venusian atmosphere (Masunaga, 2015, Masunaga et al., 2017; Nara et al., 2017) and the geocoronal emission of Earth (Kuwabara et al., 2017).

We describe here a procedure for reducing the emission power that has been frequently applied in Jupiter's auroral sciences. In this respect, some successive image extensions of EUV-L2 are temporally composited to an imaging spectrum with a temporal resolution, $\Delta T$, that is typically between $10 \mathrm{~min}$ and $10 \mathrm{~h}$ (i.e., effectively 10-600 extensions are composited). The composited imaging spectrum is spatially integrated through a region of interest, such as Jupiter's northern auroral region and the Io plasma torus, which leads to an integrated one-dimensional spectrum. The same spatial integration is applied to the detector region with no signal to estimate the radiation background count. The emission power, $P$, is then derived by spectrally integrating the product of the $1 \mathrm{D}$ spectrum with the coefficient, $D_{i}$, through wavelengths of interest (e.g., from 90 to $148 \mathrm{~nm}$ for Jupiter's aurora, which corresponds to the molecular and atomic hydrogen emission bands) as

$$
\begin{gathered}
P=\sum_{i}\left(C_{i}-G_{i}\right) D_{i}, \\
D_{i}=\frac{2 \pi R^{2} E_{i}}{A_{i} \Delta T},
\end{gathered}
$$




\section{Effective area of the EUV instrument}

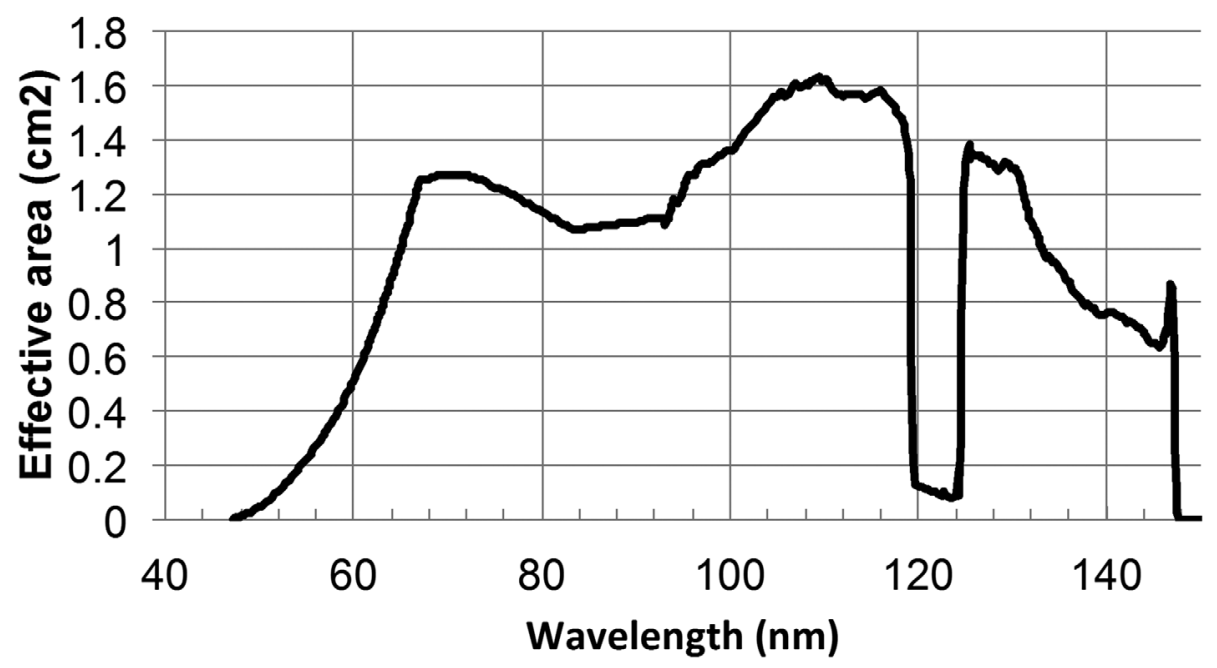

Fig. 9. Effective area of EUV instrument detector estimated from standard star white dwarf GD71 observation on 29 July, 2016. The dip at around $121.6 \mathrm{~nm}$ is due to an absence of cesium iodide photocathode, which reduced the huge number of photon counts from hydrogen Lyman alpha emission (see Yoshioka et al., 2013).

\section{EUV-CAL}

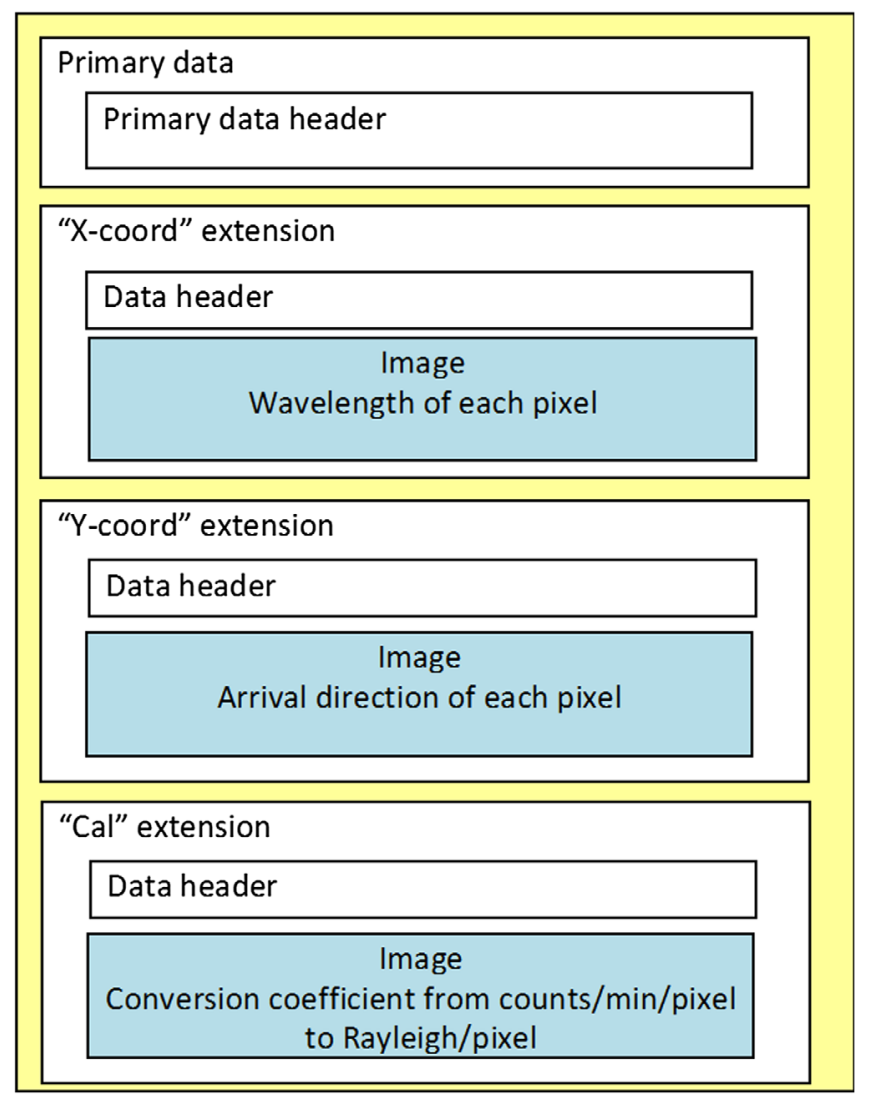

Fig. 10. FITS file structures of EUV-CAL.

where $C_{i}$ and $G_{i}$ are the spatially integrated source and background counts at the $i$ th wavelength pixel, respectively, $E_{i}$ is a photon energy at the $i$ th pixel, $A_{i}$ is the effective area

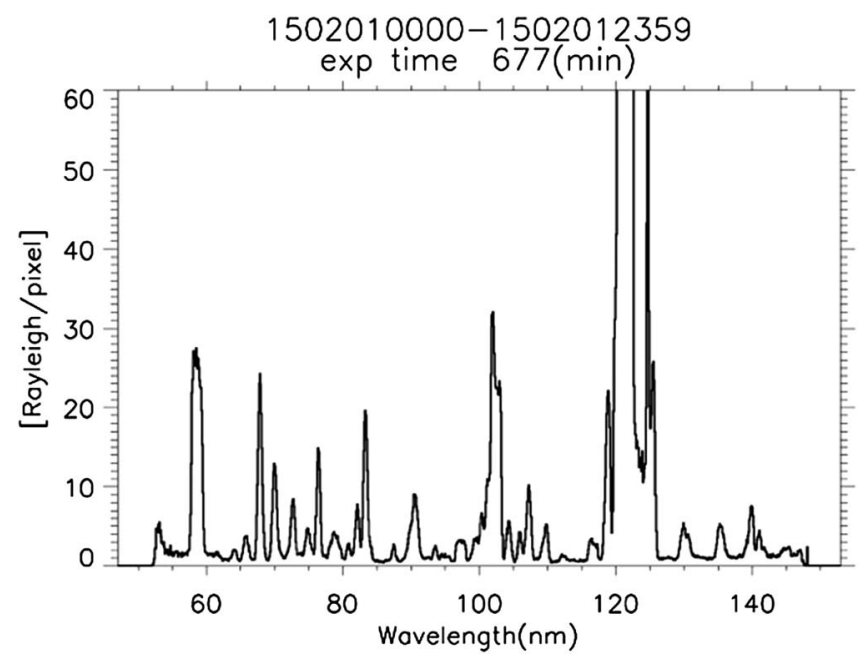

Fig. 11. An example of 1D spectrum reduced from EUV-L2 data. This spectrum is reduced from the region framed with the red broken line in Figure 7, which is at a planeto-centric distance of 3-7 Jovian radii from Jupiter. Horizontal and vertical axes show the brightness in Rayleigh/pixel and wavelength in $\mathrm{nm}$, respectively.

introduced in Section 5.2 and $R$ is the distance from the observer to the target at observation time. Here we assume an isotropic radiation source with an emission angle of $2 \pi$ steradian, where one half of a $4 \pi$ steradian isotropic emission is absorbed by the planetary body and the rest is emitted to space.

\subsection{Application of Hisaki monitoring to planetary space weather}

Based on the continuous light curve, such as that determined by 6-month monitoring of Jupiter's aurora and the Io plasma 


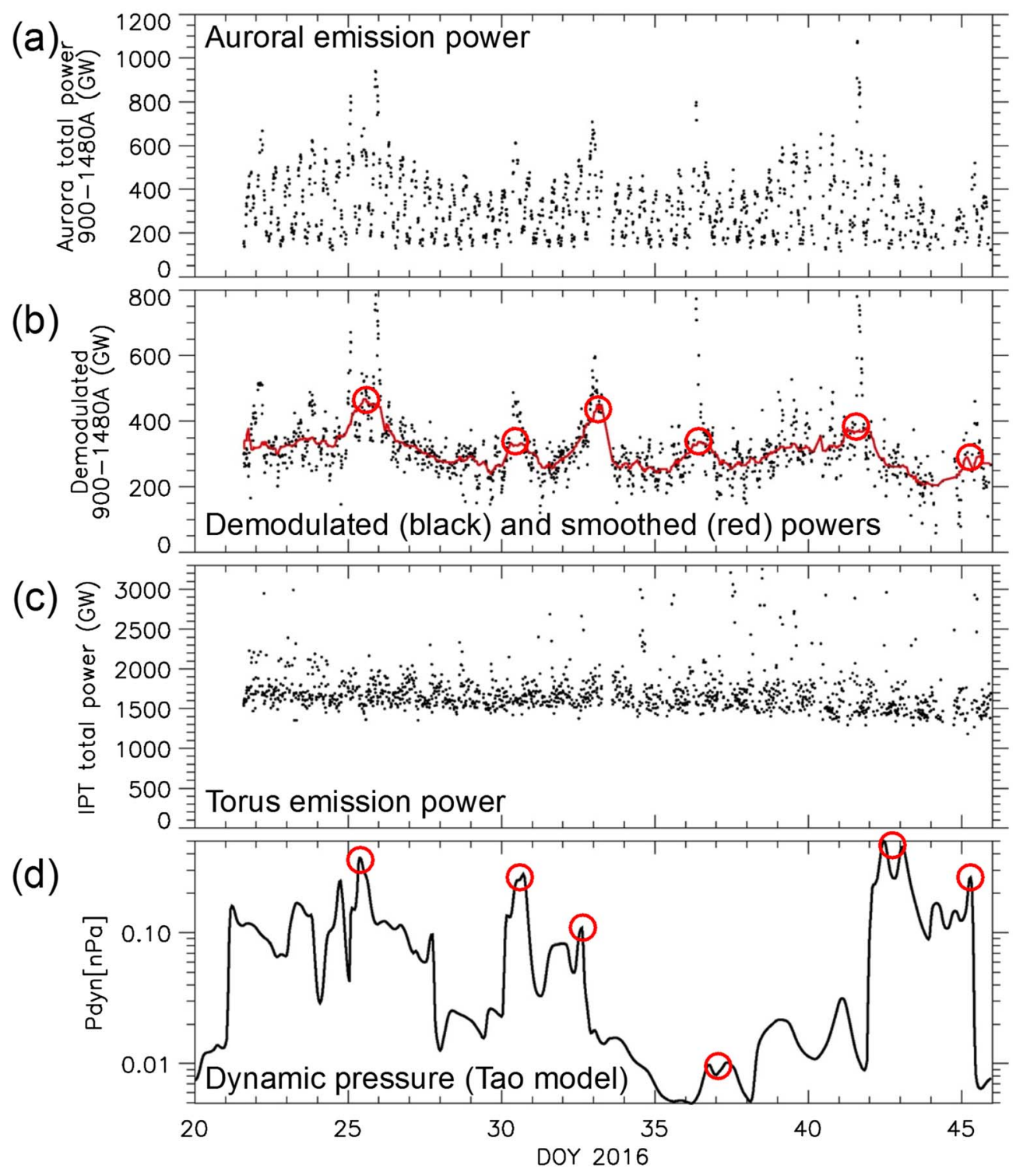

Fig. 12. Example of Hisaki monitoring of Jupiter's aurora and plasma torus observed on DOY 21-46, 2016. (a) Emission power of Jupiter's northern aurora. (b) Demodulated (black dots) and smoothed (red solid line) of auroral emission power (see Kimura et al. (2018) for the demodulation and smoothing methods). Local maxima in the smoothed power are indicated with red circles. (c) Emission power of the torus. It is of note that some data dots away from the monotonically decreasing trend are contaminated with the radiation background; this was observed when Hisaki flew through the Southern Atlantic Anomaly. (d) Solar wind dynamic pressure extrapolated from Earth's orbit by a onedimensional MHD simulation (Tao et al., 2005). Local maxima in the dynamic pressure closest to those in the smoothed auroral power are indicated by red solid circles.

torus with a temporal resolution of $10 \mathrm{~min}$ in 2015 (Kimura et al., 2018), recent Hisaki studies have revealed temporal variability in the planetary plasma and atmosphere on a timescale that ranges from tens of minutes to hundreds of days.

The responses of planetary plasma and atmosphere to variability in the solar wind are the most essential physics used to understand planetary space weather, and Hisaki monitoring data significantly contribute to this. For example, Hisaki monitoring indicated that Jupiter's auroral variability responds to the solar wind shock arrival (Kimura et al., 2015, 2016, 2017, 2018; Kita et al., 2016; Nichols et al., 2017), which suggests that the auroral current systems and any other electromagnetic processes, such as the magnetospheric tail reconnection, are modified and/or driven by conditions of the interplanetary solar wind. Simultaneously with the aurora, Hisaki monitors the plasma torus that is the dominant plasma source of Jupiter's magnetosphere. It is thus possible to discuss the variability associated with "internal" processes, such as auroral variability responding to the mass loading rate during volcanic eruptions at Io, in addition to that associated with the "external" solar wind.

Figure 12 shows an example of the response of Jupiter's aurora to the solar wind, which was observed on day of year 
(DOY) 21-46, 2016, and Figure 12a shows the emission power of the northern aurora at 90-148 nm at a temporal resolution of $10 \mathrm{~min}$; these data were reduced from EUV-L2 by the method introduced in Section 6.1. Following the analysis method of Kimura et al. (2018), the power was demodulated by subtracting a sinusoidal function fitted to the power, and the demodulated power was smoothed by a running median with a temporal window of 1 day. Demodulated and smoothed emission powers are plotted in Figure 12b as black dots and a red solid line, respectively, and local maxima in the smoothed power are marked with red circles. The emission power from the torus is shown in Figure 12c, and the solar wind dynamic pressure extrapolated from the Earth's orbit by a one-dimensional magnetohydrodynamic (MHD) simulation of Tao et al. (2005) is indicated in Figure 12d. Local maxima in the dynamic pressure closest to those in the smoothed auroral power (Fig. 12b) are indicated by the red circles. With reference to the estimation by Tao et al. (2005), uncertainty in the arrival time of the solar wind shock structure at Jupiter is estimated to be \pm 1 day for the present observation period when the Earth-Sun-Jupiter angle is approximately $30^{\circ}$.

During the observation period of Figure 12, the torus emission power was significantly weaker and stable in time compared to the active volcanic eruptions on Io observed in 2015 (Yoshikawa et al., 2017; Kimura et al., 2018), which indicates that the plasma mass loading rate was low and temporally constant. As discussed in Kimura et al. (2018), the low mass loading rate likely leads to a less frequent occurrence of the transient aurora with a typical duration of approximately $10 \mathrm{~h}$, which is associated with internally driven reconfigurations of the magnetosphere.

The smoothed emission power (red line in Fig. 12b) shows day-to-day variability on timescales longer than 1 day, where the transient aurora is successfully removed. The local maxima shown by the red circles in Figure $12 \mathrm{~b}$ are well correlated with the dynamic pressure peaks shown by the red circles in Figure 12d. The temporal difference between the auroral local maxima and the pressure peaks are less than 1 day. We conclude that the temporally smoothed auroral variability monitored with Hisaki is a useful proxy for the solar wind shock arrival to an uncertainty of within 1 day, and it is thus possible to remotely monitor solar wind variability at Jupiter based on Hisaki's auroral monitoring.

It is of note that there are some discrepancies between the aurora and solar wind: for example, the small solar wind local peaks on DOY39 and 41 are smoothed out in the auroral data. This may be partly attributed to uncertainty in the spatiotemporal evolution of the numerically simulated solar wind. A direct comparison between in-situ solar wind measurements by Juno explorer and Hisaki auroral monitoring is now being made following the method of Kita et al. (2016), and more quantitative correlations between the solar wind and Jupiter's aurora will be studied in our future work.

The transient excess of demodulated power from the smoothed power in Figure 12b can be interpreted as variability associated with the internally driven process (Kimura et al., 2015, 2017), which likely changes Jupiter's space environment via, for example, magnetospheric tail reconnection accompanying particle energization (e.g., Kronberg et al., 2007) and hot plasma injection in the inner magnetosphere
(Yoshikawa et al., 2016; Suzuki et al., 2018), which are presumably dependent on the plasma supply from Io. Hisaki observations of the aurora and torus can continuously monitor these processes, and Hisaki can thus contribute to observations of "internally driven space weather" in addition to externally (solar-wind) driven space weather.

\section{Summary}

The Hisaki satellite is the first-ever space-borne platform to monitor planets, and the authors of this study developed the ground pipeline system for Hisaki scientific data products. Millions of photon events are detected with the EUV spectrometer during 1-day observations, and these are efficiently processed in the pipeline by a computing-cost-saving algorithm and reduced to EUV-L2 imaging spectral data in the FITS format. The imaging data of FOV are also processed to the FOV-L1 FITS file, and ancillary data are processed from HK telemetry to SPICE Kernels. The EUV-L2, FOV-L1 and SPICE kernels are archived in DARTS and subsequently distributed to general users. Furthermore, higher-level data products, such as the light curve, can be reduced from EUV-L2. Recent Hisaki studies have used continuous light curve analysis to reveal planetary dynamics on timescales from tens of minutes to hundreds of days. Hisaki monitoring is an effective method for studying planetary space weather, and it is particularly useful for studying the responses of planetary environments to the solar wind and internal conditions at a high temporal resolution.

Acknowledgements. Data from the Hisaki satellite are archived at the Data Archives and Transmission System (DARTS) JAXA (https://www.darts.isas.jaxa.jp/stp/hisaki/), and users can directly access the data in DARTS. T.K. was supported by a Grant-in-Aid for Scientific Research KAKENHI (16K17812) from the Japan Society for the Promotion of Science (JSPS) and F.T. was supported by JSPS KAKENHI Grant Numbers JP26400476 and JP17H02965. The authors acknowledge the support of the International Space Science Institute (ISSI), as this study was discussed within the ISSI International Team "The influence of Io on Jupiter's magnetosphere". Furthermore, this work was supported by JSPS and MAEDI under the Japan-France Integral Action Program (SAKURA). The editor thanks two anonymous referees for their assistance in evaluating this paper.

\section{References}

Badman SV, Bonfond B, Fujimoto M, Gray RL, Kasaba Y, Kasahara S, Kimura T, Melin H, Nichols JD, Steffl AJ. 2016. Weakening of Jupiter's main auroral emission during January 2014. Geophys Res Lett 43: 988-997. DOI: 10.1002/2015GL067366.

Erard S., et al. 2018. VESPA: A community-driven virtual observatory in planetary science. Planet Space Sci 150: 65-85.

Fukuda S, Sawai S, Sakai S, Saito H, Nakagawa T, Tohma T, Takahashi J, Kitade K. 2008. Flexible standard bus for ISAS/JAXA small scientific satellite series. In: Proc. of the 26th International Symposium on Space Technology and Science (ISTS), 2008-f-17, https://archive.ists.or.jp/upload_pdf/2008-f-17.pdf. 
Gray RL, Badman SV, Bonfond B, Kimura T, Misawa H, Nichols JD, Vogt MF, Ray LC. 2016. Auroral evidence of radial transport at Jupiter during January 2014. J Geophys Res 121: 9972-9984. DOI: $10.1002 / 2016 J A 023007$.

Hikida R, Yoshioka K, Murakami G, Kimura T, T Fuminori, Yamazaki A, Yoshikawa I, Iwagami N. 2018. Identification of extreme ultraviolet emission lines of the IO plasma torus observed by Hisaki/EXCEED. J Geophys Res, Accepted. DOI: 10.1002/ 2018JE005629.

Kimura T, Badman SV, Tao C, Yoshioka K, Murakami G, Yamazaki A, Tsuchiya F, Bonfond B, Steffl AJ, Masters A, Kasahara S, Hasegawa H, Yoshikawa I, Fujimoto M, Clarke JT. 2015. Transient internally-driven aurora at Jupiter discovered by Hisaki and Hubble Space Telescope. Geophys Res Lett 42: 1662-1668. DOI: 10.1002/2015GL063272.

Kimura T, Kraft RP, Elsner RF, Branduardi-Raymont G, Gladstone R, Tao C, Yoshioka K, Murakami G, Yamazaki A, Tsuchiya F. 2016. Jupiter's X-ray and EUV auroras monitored by Chandra, XMM-Newton, and Hisaki satellite. J Geophys Res Space Phys 121: 2308-2320. DOI: 10.1002/2015JA021893.

Kimura T, Nichols JD, Gray RL, Tao C, Murakami G, Yamazaki A, Badman SV, Tsuchiya F, Yoshioka K, Kita H. 2017. Transient brightening of Jupiter's aurora observed by the Hisaki satellite and Hubble Space Telescope during approach phase of the Juno spacecraft, Special Issue "Early Results: Juno at Jupiter". Geophys Res Lett 44: 4523-4531.

Kimura T, Hiraki Y, Tao C, Tsuchiya F, Delamere P, Yoshioka K, Murakami G, Yamazaki A, Kita H, Badman SV. 2018. Response of Jupiter's aurora to plasma mass loading rate monitored by the Hisaki satellite during volcanic eruptions at Io. J Geophys Res Space Phys 123. DOI: 10.1002/2017JA025029.

Kita H, Kimura T, Tao C, Tsuchiya F, Misawa H, Sakanoi T, Kasaba Y, Murakami G, Yoshioka K, Yamazaki A, Yoshikawa I. 2016. Characteristics of solar wind control on Jovian UV auroral activity deciphered by long-term Hisaki EXCEED observations: Evidence of preconditioning of the magnetosphere? Geophysical Research Letters 43: 6790-6798. DOI: 10.1002/2016GL069481.

Koga R, Tsuchiya F, Kagitani M, Sakanoi T, Yoneda M, Yoshioka K, Kimura T, Murakami G, Yamazaki A, Yoshikawa I. 2018a. The time variation of atomic oxygen emission around Io during a volcanic event observed with Hisaki/EXCEED. Icarus 299: 300-307.

Koga R, Tsuchiya F, Kagitani M, Sakanoi T, Yoneda M, Yoshioka K, Yoshikawa I, Kimura T, Murakami G, Yamazaki A. 2018b. Spatial distribution of Io's neutral oxygen cloud observed by Hisaki. J Geophys Res - Space Phys. DOI: 10.1029/ 2018JA025328.

Kuwabara M, Yoshioka K, Murakami G, Tsuchiya F, Kimura T, Yamazaki A, Yoshikawa I. 2017. The geocoronal responses to the geomagnetic disturbances. Journal of Geophysical Research 122: 1269-1276. DOI: 10.1002/2016JA023247.

Kronberg EA, Glassmeier K-H, Woch J, Krupp N, Lagg A, Dougherty MK. 2007. A possible intrinsic mechanism for the quasi-periodic dynamics of the Jovian magnetosphere. $J$ Geophys Res 112: A05203. DOI: 10.1029/2006JA011994.

Lamy L, Prangé R, Henry F, Le Sidaner P. 2015. The auroral planetary imaging and spectroscopy (APIS) service. Astron Comput 11: 138-145.

Nichols JD, Badman SV, Bagenal F, Bolton SJ, Bonfond B, Bunce EJ, Clarke JT, Connerney JEP, Cowley SWH, Ebert RW. 2017. Response of Jupiter's auroras to conditions in the interplanetary medium as measured by the Hubble Space Telescope and Juno. Geophys Res Lett 44: 7643-7652. DOI: 10.1002/2017GL073029.
Masunaga K. 2015. Periodic variations of oxygen EUV dayglow in the upper atmosphere of Venus: Hisaki/EXCEED observations. $J$ Geophys Res Planets 120: 2037-2052. DOI: 10.1002/ 2015JE004849.

Masunaga K, Seki K, Terada N, Tsuchiya F, Kimura T, Yoshioka K, Murakami G, Yamazaki A, Tao C. 2017. Dawn-dusk difference of periodic oxygen EUV dayglow variations at Venus observed by Hisaki. Icarus 292: 102-110. DOI: 10.1016/j.icarus.2016.12.027.

Murakami G, Yoshioka K, Yoshikawa I. 2010. High-resolution imaging detector using five microchannel plates and a resistive anode encoder. Appl Optics 49(16): 2985-2993. DOI: 10.1364/ AO.49.002985.

Murakami G, Yoshioka K, Yamazaki A, Tsuchiya F, Kimura T, Tao C, Kita H, Kagitani M, Sakanoi T, Uemizu K, et al. 2016. Response of Jupiter's inner magnetosphere to the solar wind derived from extreme ultraviolet monitoring of the Io plasma tours. Geophys Res Lett 43: 12308-12316. DOI: 10.1002/ 2016 GL071675.

Nakaya K, Fukuda S, Ssakai S, Yamazaki A, Uemizu K, Toriumi T, Takahashi J, Maehara M, Okahashi T, Sawa S. 2011. Development of flexible standard bus for ISAS/JAXA small scientific satellite series. In: Proc. of the 29th International Symposium on Space Technology and Science (ISTS),2011-f-19, https://archive.ists.or. jp/upload_pdf/2011-f-19.pd.

Nara Y, Yoshikawa I, Yoshioka K, Murakami G, Kimura T, Yamazaki A, Tsuchiya F, Kuwabara M, Iwagami N. 2017. Extreme ultraviolet spectra of Venusian Airglow Observed by EXCEED. Icarus 307: 207-215. DOI: 10.1016/j.icarus.2017.10.028.

Sakai S, Yamazaki A, Uemizu K, Nakaya K, Fukuda S, Sawai S, Tanaka K, Iwayama Y, Kusakawa Y, Ogura N. 2011. Utilization of a mission sensor in the attitude control loop: A cost effective approach for high pointing accuracy. In: Proc. of the 29th International Symposium on Space Technology and Science (ISTS), 2011-f-20, https://archive.ists.or.jp/upload_pdf/2011-f-20. pdf.

Suzuki F, Yoshioka K, Hikida R, Murakami G, Tsuchiya F, Kimura T, Yoshikawa I. 2018. Corotation of bright features in the Io plasma torus. J Geophys Res: Space Phys 123: 9420-9429. DOI: $10.1029 / 2018 J A 025363$.

Tao C, Kataoka R, Fukunishi H, Takahashi Y, Yokoyama T. 2005. Magnetic field variations in the Jovian magnetotail induced by solar wind dynamic pressure enhancements. $J$ Geophys Res 110: A11208. DOI: 10.1029/2004JA010959.

Tao C, Kimura T, Badman SV, André N, Tsuchiya F, Murakami G, Yoshioka K, Yoshikawa I, Yamazaki A. 2016a. Variation of Jupiter's aurora observed by Hisaki/EXCEED: 1. Observed characteristics of the auroral electron energies compared with observations performed using HST/STIS. J Geophys Res Space Phys 121: 4055-4071. DOI: 10.1002/2015JA021272.

Tao C, Kimura T, Badman SV, André N, Tsuchiya F, Murakami G, Yoshioka K, Yoshikawa I, Yamazaki A. 2016b. Variation of Jupiter's aurora observed by Hisaki/EXCEED: 2. Estimations of Auroral Parameters and Magnetospheric Dynamics. J Geophys Res Space Phys 121: 4055-4071. DOI: 10.1002/2015JA021272.

Tao C, Kimura T, Tsuchiya F, Muirakami G, Yoshioka K, Yamazaki A, Badman SV, Misawa H, Kita H. 2018. Variation of Jupiter's aurora observed by Hisaki/EXCEED: 3. Volcanic control of Jupiter's aurora. Geophys Res Lett 45: 71-79.

Tsuchiya F, Kagitani M, Yoshioka K, Kimura T, Murakami G, Yamazaki A, Nozawa H, Kasaba Y, Sakanoi T, Uemizu K. 2015. Local electron heating in the Io plasma torus associated with Io from HISAKI satellite observation. $J$ Geophys Res 120: 10317-10333. DOI: 10.1002/2015JA021420. 
Tsuchiya F, Yoshioka K, Kimura T, Koga R, Murakami G, Yamazaki A, Kagitani M, Tao C, Suzuki F, Hikida R, Yoshikawa I, Kasaba Y, Kita H, Misawa H, Sakanoi T. 2018. Enhancement of the Jovian magnetospheric plasma circulation caused by the change in plasma supply from the satellite Io. J Geophys Res 123: 6514-6532. DOI: 10.1029/2018JA025316.

Yamazaki A, Tsuchiya F, Sakanoi T, Uemizu K, Yoshioka K, Murakami G, Kagitani M, Kasaba Y, Yoshikawa I, Terada N. 2014. Field-of-View Guiding Camera on the HISAKI (SPRINT-A) Satellite. Space Sci Rev 184: 259-274. DOI: 10.1007/s11214-0140106-y.

Yoshikawa I, Yoshioka K, Murakami G, Yamazaki A, Tsuchiya F, Kagitani M, Sakanoi T, Kimura T, Kuwabara M, Fujiwara K, et al. 2014. Extreme ultraviolet radiation measurement for planetary atmospheres/magnetospheres from the Earth-orbiting spacecraft (Extreme Ultraviolet Spectroscope for Exospheric Dynamics: EXCEED). Space Sci Rev 184(1-4): 237-258. DOI: 10.1007/ s11214-014-0077-z.

Yoshikawa I, Yoshioka K, Murakami G, Suzuki F, Hikida R, Yamazaki A, Kimura T, Tsuchiya F, Kagitani M, et al. 2016. Properties of hot electrons in the Jovian inner-magnetosphere deduced from extended observations of the Io plasma torus. Geophys Res Lett 43: 11552-11557. DOI: 10.1002/2016GL070706. Yoshikawa I, Suzuki F, Hikida R, Yoshioka K, Murakami G, Tsuchiya F, Tao C, Yamazaki A, Kimura T, Kita H, et al. 2017.
Volcanic activity on Io and its influence on the dynamics of the Jovian magnetosphere observed by EXCEED/Hisaki in 2015. Earth Planets Space 69: 110. DOI: 10.1186/s40623-0170700-9.

Yoshioka K, Murakami G, Yamazaki A, Tsuchiya F, Kagitani M, Sakanoi T, Kimura T, Uemizu K, Uji K, Yoshikawa I. 2013. The extreme ultraviolet spectroscope for planetary science, EXCEED. Planet Space Sci 85: 250-260.

Yoshioka K, Murakami G, Yamazaki A, Tsuchiya F, Kimura T, Kagitani M, Sakanoi T, Uemizu K, Kasaba Y, Yoshikawa I, et al. 2014. The evidence for the global electron transportation into the Jovian inner magnetosphere. Science 345(6204): 1581-1584. DOI: 10.1126/science.1256259.

Yoshioka K, Tsuchiya F, Kimura T, Kagitani M, Murakami G, Yamazaki A, Kuwabara M, Suzuki F, Hikida R, Yoshikawa I, et al. 2017. Radial variation of sulfur and oxygen ions in the Io plasma torus as deduced from remote observations by Hisaki. J Geophys Res Space Phys 122: 2999-3012. DOI: 10.1002/2016JA023691.

Yoshioka K, Tsuchiya F, Kagitani M, Kimura T, Murakami G, Fukuyama D, Yamazaki A, Yoshikawa I, Fujimoto M. 2018. The influence of Io's 2015 volcanic activity on Jupiter's magnetospheric dynamics. Geophys Res Lett, DOI: 10.1029/ 2018 GL079264.

Cite this article as: Kimura T, Yamazaki A, Yoshioka K, Murakami G, Tsuchiya F, et al. 2019. Development of ground pipeline system for high-level scientific data products of the Hisaki satellite mission and its application to planetary space weather. J. Space Weather Space Clim. 9, A8. 